\title{
Um Estudo Sobre Grupos de Foco e o Design Studio para Criação de Oportunidades em Wearables
}

\author{
A Study of Focus Group and Design Studio to Create Opportunities in Wearables \\ Technology.
}

LIMA, Emilly Sousa de; Graduanda de Psicologia; Universidade Federal do Amazonas; SIDIA Samsung Instituto de Desenvolvimento para a Informática da Amazônia.

mostemilly@gmail.com/emilly.Is@samsung.com

\section{Resumo}

O presente artigo descreve um estudo realizado com o objetivo de criar e avaliar oportunidades para relógios inteligentes (Smartwatches). Para tanto, a técnica do design studio foi utilizada com dois grupos distintos: com e sem experiência prévia no uso dessa tecnologia. Discorreremos neste estudo a respeito dos aspectos cognitivos, relacionados ao processo criativo com base nessa técnica, como um dos fatores reforçadores da criatividade. Considerando que propor soluções para o mercado de Smartwatches ainda é um desafio, pois se compararmos com o consumo de Smartphones, apresenta pouca adoção ocupando o 4일 lugar na lista de desejos pessoais dos brasileiros ${ }^{1}$. Verificamos que o processo cognitivo relacionado à criatividade no momento da cocriação não foi minimizado pela falta da experiência prévia com o produto e que ambos os grupos alcançaram resultados consistentes que servirão de base para a continuidade e o aperfeiçoamento das soluções para Smartwatches.

Palavras Chave: Wearable; design studio; design centrado no usuário, inovação.

\section{Abstract}

The present article describes a study carried out with the objective of creating and evaluating opportunities for Smartwatches. For this, the Design studio technique was used with two distinct groups: with and without previous experience in the use of this technology. We will discuss in this study the cognitive aspects related to the creative process based on this technique as one of the reinforcing factors of creativity. Considering that proposing solutions for the Smartwatches market is still a challenge, because if we compare with the consumption of Smartphones, it still presents little adoption, occupying the 4th place in the list of personal wishes of Brazilians. We found that the cognitive process related to creativity at the time of co-creation was not minimized by the lack of previous experience with the product and that both groups achieved consistent results that will serve as a basis for the continuity and improvement of Smartwatch solutions.

Keywords: Wearable; design studio; user centered design; innovation.

"uma pesquisa realizada em 2016 pelo Site Deloitte intitulada "Global Mobile Survey" ouviu cerca de 53.000 consumidores em 31 países dos 5 continentes, no brasil foram ouvidas 2.005 pessoas em todas as regiões do país com idade de 18 a 55 anos. 


\section{Introdução}

Visando criar soluções para a tecnologia emergente de smartwatches, o objetivo deste estudo é verificar se a metodologia do design studio atua como principal promotora da criatividade em grupos de foco com dois perfis distintos, com e sem experiência prévia no uso desse tipo de tecnologia, uma vez que, o uso desse tipo de dispositivo ainda é visto apenas como um acessório de moda e não é tão usual quanto um smartphone, sobretudo pelo seu custo (KIM; SHIN; 2015, p.6-7). Por outro lado, um dos motivos pelos quais há busca significativa pela aquisição de um relógio inteligente é o desejo de cuidar do bem-estar e da saúde (KHOA, 2015, p.5), tendo uma série de funcionalidades que auxiliam e monitoram os usuários diariamente e pela comodidade de acompanhar notificações de forma rápida sem precisar tirar o celular do bolso (SILVA; RODRIGUES; MEDOLA; PASCHOARELLI; 2016, p.9). A escolha de usar a técnica do Design Studio nesta pesquisa para promoção de soluções de relógios inteligentes, foi pela possibilidade de estímulo do potencial criativo dos participantes, para dessa forma alcançar bons resultados, pois segundo Sullivan (2016, p.11) "um espaço mental é necessário para pensar, experimentar, repensar, calibrar, refazer e projetar".

O Design Studio se apresenta como uma forma de representar visualmente, estabelecendo empatia entre o usuário o produto e a empresa, corroborando pensamento intuitivo com percepção, memória e estimulando a auto-eficácia ${ }^{2}$, pois os participantes são desafiados a não apenas buscarem soluções, mas a justificarem suas ideias, representando-as e defendendo os seus pontos de vista, trazendo suas experiências e percepções, e consequentemente diminuindo possíveis riscos ao propor novos caminhos para a tecnologia vestível. A prática do design studio desmistifica o mito e a distinção valorativa do gênio criativo, detentor de ideias iluminadas pois a criatividade decorre de fatores culturais, é inata a todos os sujeitos, podendo ser estimulada por fatores psicossociais, não sendo característica de alguns indivíduos mais favorecidos, ela pode ser treinada e reforçada (OSTROWER, 1977). Por meio disso, a problemática central desta investigação é observar se a ausência da experiência direta com o produto impacta no processo criativo no momento da atividade de co-criação. Além disso, buscamos avaliar se as ideias geradas pelos participantes, serviria como base para a criação e o aperfeiçoamento de soluções para o mercado de relógios inteligentes.

Investigar a criatividade em grupos de foco, implica pensarmos em modos de como o sujeito age, toma decisões, pensa e se comporta, por meio de aspectos cognitivos, ou seja, a relação da percepção, atenção, memória, linguagem e aprendizado, por meio de fatores emocionais e psicossociais que influenciam no seu comportamento ao imaginar, criar e representar (WECHSLER, 1998, p.1). Em outras palavras, os sujeitos trazem suas experiências e no momento da co-criação, projetam suas expectativas para o aprimoramento da tecnologia. Mesmo que os participantes não possuam o produto, é visível que os mesmos conseguem correlacionar modelos mentais de "relógio inteligente" com conhecimentos baseados em experiências indiretas com o produto, pois as novidades tecnológicas acabam chegando de forma implícitas ou explícitas ao indivíduo através dos compartilhadores de conteúdo em mídias sociais (DROGE, STANKO,POLLITTE, 2009, p.16).

2 teoria social de Albert Bandura (1986) da qual diz respeito à capacidade do sujeito em acreditar em suas habilidades e desenvolvê-las a partir da crença que ele tem em si mesmo. 
Ao iniciar uma pesquisa com usuários, é comum pensarmos de início quem serão os participantes, quais são os seus costumes básicos e nível de conhecimento em determinada tecnologia, ou seja, qual é o perfil esperado para poder partir para o levantamento de uma metodologia, e assim, conseguir resultados que apoiam a tomada de decisão. Por vezes, as limitações nos impedem de encontrar os perfis esperados, sendo necessário buscar meios que não prejudiquem o desígnio da pesquisa. Isto posto, procuramos investigar se trabalhando com dois grupos de foco com perfis diferentes influenciava no resultado e no potencial criativo desses grupos. Veremos os principais resultados obtidos e os conceitos levantados por meio dessa metodologia, dialogando com o design centrado no usuário e aspectos psicológicos que norteiam o processo cognitivo da criatividade, verificando o método do design studio como promotor e fortalecedor da imaginação e criação de propostas para tecnologias emergentes de smartwatch.

\section{Metodologia}

A pesquisa foi qualitativa, ocorrendo em treze sessões com usuários num período de um mês, a duração dos encontros era de uma hora e meia aproximadamente. Os construtos principais da investigação para cada conceito foram: funcionalidades, interface e cenários de uso.

Um questionário online foi criado primeiramente para o recrutamento dos possíveis participantes, contendo vinte questões divididas entre perguntas discursivas e de múltipla escolha para mapear e entender os hábitos e as expectativas de melhoria no uso de relógios e pulseiras inteligentes (Smartwatches e Bands), com o intuito de selecionar o grupo de usuários com experiência. Havia também nesse questionário, dez itens sócio demográficos, e itens que contemplavam pessoas que não tinham o dispositivo, contudo manifestavam interesse em adquirir no futuro, o principal propósito era de identificarmos o grupo de foco sem a experiência prévia com a tecnologia, ou seja, eram pessoas que já apresentavam interesse pelo uso de vestíveis.

A amostra no geral foi de vinte e nove pessoas selecionadas, destes, oito eram mulheres e vinte e um eram homens com idade entre 19 e 40 anos. A maior parte dos participantes trabalhava, estudava e tinham hábitos em comum: ambos os grupos pesquisavam sobre novidades tecnológicas em sites, blogs e revistas, além de que, eram ativos na aquisição de novas tecnologias.

Após a seleção dos dois grupos de foco, com e sem a experiência prévia no uso da tecnologia, (grupo A e grupo B) partíamos para a co-criação de dois conceitos, um voltado para lembretes de afazeres diários, e outro voltado para o aprendizado de vocabulários de línguas estrangeiras. Em ambos os grupos, por meio de um pequeno mapa de experiência, exploramos categorias gerais da experiência do usuário, como: funcionalidades, interface e cenários de uso que eram sondados durante a co-criação a partir de suas criações. Partimos da premissa de que a principal vantagem do design studio é criar um espaço de possibilidades criativas.

No momento da atividade de ideação, iniciamos a pesquisa com uma breve conversa exploratória a fim de perceber perfis e hábitos, em seguida, era solicitado que eles listassem as principais dificuldades ao aprender determinados vocabulários idiomáticos e ao lembrar de atividades corriqueiras do dia-a-dia, com o objetivo de prepará-los para as próximas etapas do processo e identificar os pontos chaves para a criação. Em seguida, havia a representação das ideias obtidas num papel em branco com desenhos de smartwatch, a demonstração e crítica das ideias 
por todos do grupo e por fim, os participantes eram convidados a juntar suas ideias para criar em conjunto um "produto final". Cada um escolhia as funcionalidades do outro, ou o grupo decidia entre si qual ideia seria mais vantajosa utilizar, a tomada de decisão partia deles próprios.

Era utilizado razoável número de canetas coloridas, várias folhas de papel ofício, cronômetro para controle das tarefas e um modelo pré-definido de smartwatch no papel, para facilitar a ideação. A figura abaixo ilustra os materiais utilizados, pelos grupos para desenvolverem e representarem suas ideias:

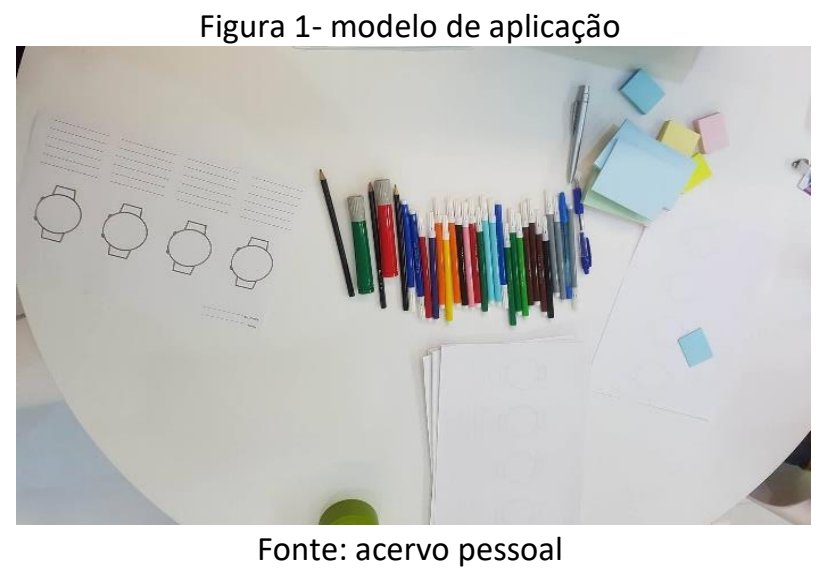

Para mitigar as limitações que o desconhecimento do dispositivo poderia suscitar, mostramos três modelos de smartwatches e bands (relógio e pulseira inteligente) para que os integrantes dos grupos pudessem experimentar cada um desses dispositivos.

Os produtos utilizados na pesquisa foram: um Samsung Gear S3, um Samsung Gear S2 e uma Smartband Fit 2. Os smartwatches da Samsung, Gear S2 e Gear S3 possuem conectividade bluetooth, sensores que auxiliam no monitoramento da saúde no dia-a-dia do usuário, como: podômetros, frequência cardíaca e acelerômetro, além de possuir funcionalidades que ajudam na comodidade ao acompanhar notificações, controle de faixa musical e responder mensagem de texto de forma instantânea. Já a pulseira inteligente Samsung Gear Fit 2, é mais direcionada para o controle de atividades físicas, contendo também: conexão via Bluetooth com o smartphone, sensores de frequência cardíaca, acelerômetro e podômetro, possuindo apenas algumas limitações de funcionalidades comparado ao smartwatch.

Como mencionado previamente, em ambos os grupos a maioria dos participantes apresentava interesse por pesquisar e consumir frequentemente produtos ou serviços tecnológicos, ou seja, os participantes que não possuíam experiência com o produto, são os considerados early adopters "visionários" pessoas que têm contato com novas tecnologias e buscam conhecer novos produtos adquirindo-os antes da maioria dos consumidores que são os considerados "pragmáticos" (MOORE, A. 2014). Segundo Chau e Hui (1998, p.2) conforme exposto por Rogers (1983) "a adoção envolve cinco etapas básicas (conscientização, interesse, avaliação, julgamento e finalmente adoção) ", observamos que os participantes sem a experiência prévia, estavam na segunda etapa, a de adoção, pois já sabiam do que se tratava e tinham interesse na aquisição da tecnologia, todavia ainda faltava as demais etapas para a adoção definitiva ao produto. 


\section{Resultado e Discussões}

Conforme exposto, a metodologia do design studio foi aplicada com o intuito de verificar propostas para dois conceitos, sendo eles: um voltado para o controle do tempo em atividades do dia-a-dia e outro voltado para o aprendizado de vocabulários em línguas estrangeiras. Os principais resultados obtidos podem ser verificados nas tabelas a seguir:

Tabela 1 - Grupo A e B, lembretes de atividades do dia-a-dia

\begin{tabular}{ccc} 
Funcionalidades & Interface & Cenários de uso \\
\hline $\begin{array}{c}\text { Recurso de voz/interação por } \\
\text { voz } \\
\text { Inteligência Artificial }\end{array}$ & Ícones pré-definidos & Trabalhando \\
& $\begin{array}{c}\text { Indicadores prioritários de } \\
\text { alarme }\end{array}$ & Trabalhando \\
\hline & Fonte: o autor
\end{tabular}

Fonte: o autor

Tabela 2 - Grupo A e B, Aprendizado de vocabulários em línguas estrangeiras

\begin{tabular}{ccc} 
Funcionalidades & Interface & Cenários de uso \\
\hline $\begin{array}{c}\text { Recurso de voz/interação } \\
\text { por voz } \\
\begin{array}{c}\text { Recurso de voz/interação } \\
\text { por voz }\end{array}\end{array}$ & Vocabulários com imagens & Interagindo com pessoas \\
& Inteligência artificial & Assistindo filmes e séries \\
\hline
\end{tabular}

No conceito de afazeres diários, no item ícones pré-definidos, os participantes sugeriram ícones associados a atividades momentâneas, como por exemplo: uma panela que ao ser selecionada indica que em 30 minutos o feijão estará pronto. No item inteligência artificial, recomendaram o reconhecimento de ambientes que envia alerta ao identificar algum acontecimento estranho (ex. mudança de temperatura pode indicar incêndio), em interface propuseram indicadores prioritários que reconhecem automaticamente através de movimento do braço e sinaliza por vibração. Luz e cores como indicadores de tempo decorrido da tarefa.

Já com relação ao projeto de aprendizado em vocabulários em línguas estrangeiras, no item funcionalidades, recurso de voz/interação por voz, significa ouvir e treinar pronúncias e expressões idiomáticas e interação direta por input e output através de inteligência artificial. Em interface, sugeriram interação direta por meio de inteligência artificial, ou seja, receber vocabulários de acordo com o contexto do local ou reconhecimento de alguma atividade que está sendo executada no momento. 
É possível visualizar pequenas diferenças entre os grupos A e B com relação às categorias investigadas na proposta voltada para lembretes de afazeres diários, e similaridades no que tange o item cenários de uso (trabalhando), além disso, foi percebida uma convergência de resultados com relação à proposta de línguas estrangeiras nas categorias de investigação acima (recurso de voz/interação por voz). No geral, é possível verificar também que há um anseio por inteligência artificial na maior parte das categorias investigadas (reconhecimento de ambientes, aviso relacionado a mudanças de temperatura ou distância, por exemplo, e integração de relógios inteligentes com outros elementos da casa ou do trabalho por meio de IOT "internet of things", também foram mencionados) e o desejo de um dispositivo que funcione de maneira mais independente, visto há um grande volume de limitações de funcionamentos que promovem frustrações aos usuários, como por exemplo: o consumo de bateria (SILVA G. et al, 2016 p.9).

Por meio disto, os resultados obtidos pelos dois grupos (A e B), foram satisfatórios, apesar de alguns resultados serem divergentes entre si, a qualidade das propostas encontradas nos grupos de foco não foram minimizadas, sendo avaliadas como consistentes. Segundo Daalhuizen (2014) apud ZAVADIL, P. (2016) "[...] estratégias e mecanismos podem estimular o pensamento criativo, sobretudo o pensamento por analogias, facilitando a ativação de conhecimentos armazenados na memória e o estabelecimento de relações para o desenvolvimento de soluções", ter pessoas de diferentes perfis mas que se assemelham em determinados aspectos, como: interesse tecnológico, por exemplo, talvez seja um dos fatores na qualidade de resultados e potencial criativo para esse tipo de tecnologia, pois os participantes faziam relações com outros dispositivos e trocavam experiências entre eles ao construírem em conjunto uma ideia final. Além do que, o grupo sem a experiência prévia foi recrutado por meio do questionário online onde afirmavam previamente que tinham interesse em adquirir o produto nos próximos seis meses, ou seja, podemos deduzir que eles já haviam buscado conhecer sobre a ficha técnica e o preço do produto.

Sullivan (2016, p.14) afirma que "... as interações do grupo devem levar a uma maior criatividade, à medida que as pessoas se baseiam nas ideias dos outros", isso leva-nos a pensar também que o aspecto social de interação e de compartilhamento de ideias na metodologia do design studio, ratifica ainda mais os aspectos cognitivos e de criatividade.

Esse estudo identificou que ter perfis dissemelhantes com relação à experiência de uso, não interfere nos resultados, pois a convergência de ideias pode ser diferente, mas a capacidade criativa é a mesma e até maior, por vezes, com não usuários da tecnologia investigada, pois estas pessoas por não terem conhecimento das limitações que existem no uso do dispositivo, acabam elaborando e projetando maior potencialidades imaginativas e representativas, se compararmos com os usuários da tecnologia. Sendo assim observado, constatamos que ao propor soluções para o mercado de tecnologias emergentes.

Ter usuários com experiência no uso do produto é vantajoso pelo fato deles articularem os limites da tecnologia, por outro lado, não usuários tendem a ter alto potencial criativo e ideias mais à frente do que é possível no cenário atual do produto, se levarmos em consideração a dificuldade de encontrar perfis com experiência em determinado dispositivo, dependendo do objetivo da pesquisa ao propor soluções inovadoras é de considerável relevância dar voz à perfis como estes encontrados neste estudo. 


\section{Conclusão}

Logo, é compreendido a partir desse experimento que a criatividade ao aplicar a metodologia do design studio não foi minimizada ao ter usuários sem a experiência prévia com o uso de smartwatches, pois eles fazem correlações com outros tipos de tecnologias e experiências, como por exemplo: aplicativos de lembretes e de aprendizagem de vocabulários similares aos conceitos investigados aqui e desenvolvidos em outras plataformas (smartphones, tablets, PC), do qual eles mencionavam no momento da atividade. Ademais, o método aplicado na pesquisa acentua a interação social e o compartilhamento de ideias, possibilitando maior criatividade ao gerar soluções.

Os fatores de conhecimento indireto e o interesse em aquisição ao produto num breve período de tempo, verificados no momento do recrutamento dos grupos sem a experiência, bem como o conhecimento empírico baseado em modelos mentais obtidos através de outras plataformas e redes de compartilhamento de informações, também se configuram como um dos fatores reforçadores no momento de idealizar soluções para os dispositivos. Também é possível inferir que os usuários no momento da co-criação tendem a propor soluções mais acuradas de interface e experiências (ex. recurso de voz e interação por voz), em contrapartida, os não usuários da tecnologia tendem a ter maior desempenho imaginativo e criativo para além do que é possível na tecnologia (ex. inteligência artificial).

A lacuna da ausência de experiência prévia no uso de smartwatch não diminuiu os aspectos cognitivos da co-criação (percepção, memória, aprendizagem e representação). O conhecimento e a experiência de uso são aspectos essenciais no processo de construção de oportunidades em tecnologias inovadoras. Podemos investigar em futuras pesquisas se o mesmo acontece em processos de criação de oportunidades para outros tipos de tecnologias emergentes, sobretudo, validar questões de conhecimento indireto versus experiência prévia, pois investigar modos de comportamento humano - computador é diminuir possíveis riscos ao dar início a uma proposta inovadora.

\section{Agradecimentos}

Meus agradecimentos ao SIDIA e seus times, singularmente ao time de UX \& Design por possibilitar que essa e outras pesquisas sejam levadas à construção do conhecimento em todo território nacional e internacional e à Faculdade de Psicologia da Universidade Federal do Amazonas e meus queridos mestres e amigos. 


\section{Referências}

NORMAN, D. A. The design of everyday things. Ed. 2002 New York: Basic Books, 2002.

SULLIVAN, B. The design studio method - creative problem solving with UX sketching. Focal Press: Burlington, 2016.

OSTROWER F. Criatividade e processos de criação. Editora Vozes:Rio de Janeiro, 1977.

WECHSLER S. Estilos de pensar e criar: impactos nas áreas educacional e profissional. Universidad de Palermo, Buenos Aires, 2006.

WESCHESLER S. Avaliação multidimensional da criatividade: uma realidade necessária. V.2 n.2, 1998 Pontífica Universidade Católica de Campinas, Psicologia Escolar e Educacional. On-line version ISSN 2175-3539

ZAVADIL, P.; SILVA, R.; TSCHIMMEL, K. Modelo teórico do pensamento e processo criativo em indivíduos e em grupos de design. Design e Tecnologia, [S.I.], v. 6, n. 12, p. 1-20, dez. 2016. ISSN 2178-1974. Disponível em: <https://www.ufrgs.br/det/index.php/det/article/view/380>. Acesso em: 27 jun. 2017. doi: http://dx.doi.org/10.23972/det2016iss12pp1-20.

DAALHUIZEN, Jaap. Method Usage in Design: How methods function as mental tools for designers. 2014. 235 f. PhD (thesis). Delft University of Technology, Netherlands, Delf.

Khoa, Tran. Wearables smart technologies: new era of technology. 2015. 65 f. Degree Programme in Innovative Business Services (thesis). Lepland University of Applied Sciences. Finland.

SOBRINHO, C. A relação entre reflexividade, normas, elaboração da informação e criatividade do grupo: uma análise a partir da perspectiva de grupos como processadores de informação. 2016. 181

f. Programa de Pós-Graduação em Administração de Empresas, Universidade Presbiteriana Mackenzie, São Paulo - SP.

$\mathrm{CHUAH}, \mathrm{S} . \mathrm{H}$. W. et al. Wearable technologie: the role of usefulness and visibility in smartwatch adoption. Computers in Human Behavior. v.n.1 p. 276-284, jan./jul., 2016.

KRANZ, G., FERSCHA, A. Size Does Matter - Positioning on the Wrist A Comparative Study: SmartWatch vs. SmartPhone. In: The Third IEEE International Workshop on Sensing Systems and Applications Using Wrist Worn Smart Devices, 3., 2017, Kona, HI, USA. 2017.

KIM K. J., SHIN D. H. An acceptance model for smart watches-Implications for the adoption of future wearable technology, Internet Research, v. 25 Iss 4 p. 527 - 541, 2015. 
DROGE, C., STANKO, M., POLLITTE, W. Lead Users and Early Adopters on the Web: The Role of New Technology Product Blogs. The Jornal of Product Innovation Management, v. 27, p.66 - 82, dec./jan., $2009 / 2010$.

CHAU, P. K., HUI, K. L., Identifying early adopters of new IT products: A case of Windows 95. Information and Management, School of Business and Management, The Hong Kong University of Science and Technology, Hong Kong, v. 33, p. 225-230, feb./dec. 1997.

SILVA, Gustavo de Andrade; RODRIGUES, Sérgio Tosi; MEDOLA, Fausto Orsi; PASCHOARELLI, Luis Carlos; "AVALIAÇÃO QUALITATIVA DA USABILIDADE E MOTIVAÇÕES DE USUÁRIOS DE RELÓGIOS INTELIGENTES", p. 1104-1113. In: 10 Congresso Internacional de Ergonomia Aplicada [Blucher Engineering Proceedings, v.3 n.3]. São Paulo: Blucher, 2016. ISSN 2357-7592, DOI 10.5151/engproconaerg2016-6834

PANDELO, Helcio de Macedo. IoT e Dispositivos Vestíveis Aplicados à Área da Saúde. 2016. $15 f$. Monografia apresentada Centro Universitário Eurípedes de Marilha, para obtenção do grau de bacharel em sistema da informação.

LIU X. et al. Characterizing Smartwatch Usage in the Wild, p.385-398. In: Proceedings of the 15th Annual International Conference on Mobile Systems, Applications, and Services. New York, USA June 19-23, 2017. ISBN: 978-1-4503-4928-4 doi>10.1145/3081333.3081351 